\title{
PREVALÊNCIA DE SINTOMAS DEPRESSIVOS E CONDIÇÕES DE SAÚDE EM IDOSOS ATENDIDOS NA ATENÇÃO PRIMÁRIA À SAÚDE
}

\author{
PREVALENCE OF DEPRESSIVE SYMPTOMS AND \\ HEALTH CONDITIONS IN THE ELDERLY TREATED \\ IN PRIMARY HEALTH CARE
}

\section{PREVALENCIA DE SÍNTOMAS DEPRESIVOS Y CONDICIONES DE SALUD EN ANCIANOS TRATADOS EN ATENCIÓN PRIMARIA DE SALUD}

\author{
Evanilza Maria Marcelino ${ }^{1}$ \\ Priscila Maria de Castro Silva ${ }^{2}$ \\ Fabíola de Araújo Leite Medeiros ${ }^{3}$ \\ Josefa Raquel Luciano da Silva ${ }^{4}$ \\ Ricardo Alves de Olinda ${ }^{5}$ \\ Ana Claudia Torres de Medeiros ${ }^{6}$
}

Como citar este artigo: Marcelino EM, Silva PMC, Medeiros FAL, Silva JRL, Olinda RA, Medeiros ACT. Prevalência de sintomas depressivos e condições de saúde em idosos atendidos na Atenção Primária à Saúde. Rev baiana enf. 2022;36:e45832.

Objetivo: analisar a prevalência de sintomas depressivos e as condições de saúde em idosos atendidos na Atenção Primária à Saúde. Método: pesquisa observacional do tipo transversal, de abordagem quantitativa, realizada com 130 idosos de um município brasileiro. Coleta de dados ocorreu entre novembro de 2019 e março de 2020, utilizando Escala de Depressão Geriátrica (GDS-15) e questionário, contemplando dados sociodemográficos. Resultados: prevalência de sintomas depressivos presentes em 78 idosos $(63,1 \%)$. As variáveis independentes associadas foram: sexo feminino, idade entre 60 e 69 anos, renda mensal de 1 a 3 salários-mínimos, maior que nove anos de escolaridade, domínio mental e físico com risco, com mais de três doenças autorreferidas e mais de cinco medicamentos utilizados. Conclusão: a alta prevalência de sintomas depressivos na população idosa estudada requer investimento em ações de prevenção, atentando para a necessidade de práticas que promovam o envelhecimento ativo e a satisfação com a vida.

Descritores: Saúde do Idoso. Envelhecimento. Depressão. Atenção Primária à Saúde. Enfermagem.

Objective: to analyze the prevalence of depressive symptoms and health conditions in the elderly treated in Primary Health Care. Method: cross-sectional observational research, with a quantitative approach, carried out with

\footnotetext{
Enfermeira. Pesquisadora Independente. Equador, Rio Grande do Norte, Brasil. isamaria.ufcg@gmail.com. https://orcid.org/0000-0003-0589-2290.

Enfermeira. Doutora em Enfermagem. Professora da Universidade Federal de Campina Grande. Campina Grande, Paraíba, Brasil. https://orcid.org/0000-00020344-8367.

Enfermeira. Doutora em Enfermagem. Professora da Universidade Estadual da Paraíba. Campina Grande, Paraíba, Brasil. https://orcid.org/0000-0002-0834-I I55.

4 Enfermeira. Residente em Atenção Básica e Saúde da Família. Joboatão dos Guararapes, Pernambuco, Brasil. https://orcid.org/0000-0003-1791-5541.

5 Estatístico. Doutor em Estatística. Professor da Universidade Estadual da Paraíba. Campina Grande, Paraíba, Brasil. https://orcid.org/0000-0002-0509-8428.

Enfermeira. Doutora em Enfermagem. Professora da Universidade Federal de Campina Grande. Campina Grande, Paraíba, Brasil. https://orcid.org/0000-0002$3695-9745$.
} 
130 elderly people from a Brazilian municipality. Data collection occurred between November 2019 and March 2020, using the Geriatric Depression Scale (GDS-15) and a questionnaire, including sociodemographic data. Results: prevalence of depressive symptoms present in 78 elderly (63.1\%). The associated independent variables were: female gender, age between 60 and 69 years, monthly income from 1 to 3 minimum wages, greater than nine years of schooling, mental and physical domain at risk, with more than three self-reported diseases and more than five medications used. Conclusion: the high prevalence of depressive symptoms in the elderly population studied requires investment in prevention actions, noting the need for practices that promote active aging and life satisfaction.

Descriptors: Health of the Elderly. Aging. Depression. Primary Health Care. Nursing.

Objetivo: analizar la prevalencia de sintomas depresivos y condiciones de salud en ancianos tratados en Atención Primaria de Salud. Método: investigación observacional transversal, con enfoque cuantitativo, realizada con 130 ancianos de un municipio brasileño. La recolección de datos ocurrió entre noviembre de 2019 y marzo de 2020, utilizando la Geriatric Depression Scale (GDS-15) y un cuestionario, incluyendo datos sociodemográficos. Resultados: prevalencia de sintomas depresivos presentes en 78 ancianos (63,1\%). Las variables independientes asociadas fueron: sexo femenino, edad entre 60 y 69 años, ingresos mensuales de 1 a 3 salarios minimos, mayor a nueve años de escolaridad, dominio mental y físico en riesgo, con más de tres enfermedades autoinformadas y más de cinco medicamentos utilizados. Conclusión: la alta prevalencia de síntomas depresivos en la población anciana estudiada requiere inversión en acciones de prevención, señalando la necesidad de prácticas que promuevan el envejecimiento activo y la satisfacción con la vida.

Descriptores: Salud del Anciano. Envejecimiento. Depresión. Atención Primaria de Salud. Enfermería.

\section{Introdução}

O fenômeno do alongamento do tempo de vida verificou-se, inicialmente, nos países desenvolvidos, porém, mais recentemente, é nos países em desenvolvimento que o envelhecimento da população tem crescido de forma mais acentuada. No Brasil, de acordo com as previsões, enquanto o número de idosos, entre 1950 e 2025, aumentará em 15 vezes, na população total crescerá 5 vezes ${ }^{(1)}$.

Seguindo a transição demográfica da população, percebe-se que o Brasil tem envelhecido de maneira rápida e intensa, com a maioria dos idosos apresentando baixo nível socioeconômico e alta prevalência de doenças crônicas não transmissíveis (DCNT). Este fato ocasiona uma sobrecarga substancial para a economia nacional, especialmente pelos gastos com aposentadoria e recursos médicos. Além disso, essas condições constituem as principais causas de mortalidade $\mathrm{e}^{(2-3)}$.

Assim, nos últimos anos, com o envelhecer da população, a temática do envelhecimento tem ganhado relevância, especialmente pelo consequente aumento de doenças crônicas notificadas nessa população, incluindo as doenças psiquiátricas. Diante desse contexto, a depressão no idoso surge como um dos destaques que merece atenção especial ${ }^{(4)}$.

Considerando que o cuidado em saúde abrange aspectos biopsicossociais, a Atenção Primária em Saúde (APS) é um importante cenário no cuidado ao idoso. Por ser preferencialmente uma porta de entrada referencial aos serviços de saúde, para atuação na promoção de saúde mental, destaca-se o papel das equipes no desenvolvimento de ações de rastreio, encaminhamento e monitoramento de usuários com sintomas de depressão ${ }^{(5)}$.

Ressalta-se que a depressão pode ser desencadeada por fatores biológicos, a exemplo da genética, que é significativa no desenvolvimento de um quadro depressivo. Além disso, fatores psicológicos causam perda da autonomia e agravamento de quadros patológicos preexistentes no idoso, assim como fatores sociais, que interferem na capacidade funcional do autocuidado e nas relações sociais ${ }^{(6-8)}$.

Diante disso, considera-se relevante obter dados que possam desencadear novas reflexões e propostas de intervenção relacionadas à atenção ao idoso, principalmente no tocante à saúde mental, pelo fato de poderem condicionar 
sofrimento e diminuição da qualidade de vida das pessoas afetadas ${ }^{(0-10)}$.

O problema de pesquisa debruçou-se, sobretudo, na identificação dos fatores biopsicossociais que perpassaram a depressão de idosos na cidade de Equador (RN), como indicador do caminho ideal para o desenvolvimento de ações preventivas eficazes, incluindo a descoberta de novas possibilidades de atuação do setor de saúde do município em questão.

Dessa forma, esta pesquisa teve como objetivo analisar a prevalência de sintomas depressivos e as condições de saúde em idosos atendidos na Atenção Primária à Saúde.

\section{Método}

Estudo de investigação epidemiológica do tipo observacional, de corte transversal e abordagem quantitativa. A amostra foi realizada pelo método conglomerados entre os idosos atendidos nas Unidades Básicas de Saúde (UBS) do município Equador, estado do Rio Grande do Norte. Os dados foram coletados entre novembro de 2019 e março de 2020, após a aprovação do Comitê de Ética em Pesquisa do Hospital Universitário Alcides Carneiro (HUAC), sob Parecer $n^{\circ} 3.155 .477$, em conformidade com os aspectos éticos e científicos preconizados pela Resolução no 466/2012 do Conselho Nacional de Saúde (CNS) sobre pesquisas que envolvem seres humanos ${ }^{(11)}$.

De acordo com o contingente total de 692 idosos do município, o cálculo amostral resultou em um n equivalente a 182 participantes, considerando o nível de confiança de 95\% e erro amostral de 5\%. Excluindo as perdas, recusas amostrais e levando em consideração a pandemia por Covid-19, que dificultou a execução da coleta, participaram do estudo 130 idosos selecionados com base no modelo de amostragem probabilística sistemática.

Dessa forma, foram escolhidas as três Unidades Básicas de Saúde (UBS) do município, que atendiam os 692 idosos da zona urbana do município. Realizada a distribuição por unidade básica, os domicílios foram visitados com a participação de Agentes Comunitários de Saúde (ACS).
Os critérios de inclusão utilizados foram: indivíduos com idade igual ou maior que 60 anos, acompanhados pelas equipes de saúde das respectivas UBS durante o período da coleta de dados. Foram excluídos os usuários das unidades que possuíam diagnóstico de demência ou alteração cognitiva que impossibilitassem ou dificultassem o preenchimento do instrumento.

Para avaliação dos aspectos cognitivos, foi aplicado o Mini Exame do Estado Mental (MEEM) traduzido e validado no Brasil ${ }^{(12)}$. Este instrumento é composto de questões agrupadas em sete categorias, com um escore total de 0 a 30 pontos.

Os idosos incluídos na pesquisa, após o MEEM, foram questionados acerca do perfil socioeconômico, das condições e dos hábitos de vida. O instrumento de coleta aplicado aos entrevistados foi desenvolvido pelos autores. As variáveis independentes foram: informações sociodemográficas (faixa etária, sexo, escolaridade, renda, situação conjugal e moradia, serviço de saúde utilizado); comportamentos e condições normativas de saúde (hábitos tabagista e/ou etilista, nível de atividade física e presença de doenças crônicas). Também foi perguntado sobre doenças autorreferidas (asma/bronquite, hipertensão arterial sistêmica, hipercolesterolemia, reumatismo entre outros) e uso de medicamentos.

Por fim, aplicou-se a Escala de Depressão Geriátrica Abreviada (GDS-15) ${ }^{(13)}$, em sua versão $\operatorname{adaptada}^{(14)}$. Este instrumento de rastreio de sintomas depressivos em idosos é composto de 15 perguntas com respostas binárias (sim/não) a respeito de como a pessoa tem se sentido durante a última semana.

Os itens avaliam a satisfação de vida pessoal, o estado de humor, a irritabilidade, os pensamentos negativos, a felicidade, a capacidade de solucionar problemas, o interesse em sair de casa e fazer coisas novas ou coisas que já gostava, em detrimento a ficar em casa sozinha, a sensação de inutilidade, de energia e de esperança e sentir-se bem ou pior que as outras pessoas. A versão brasileira da GDS-15 é o instrumento mais indicado para esse tipo de avaliação, por possuir 
índices de sensibilidade de $85,4 \%$ e especificidade de $73,9 \%{ }^{(14)}$.

Para análise e organização dos dados da pesquisa, utilizou-se a estatística descritiva, com apresentação de frequências simples, absolutas e percentuais para as variáveis categóricas e posterior organização dos resultados em tabelas. $\mathrm{Na}$ sequência, aplicou-se o teste de aderência de qui-quadrado, para verificar a adequabilidade do modelo probabilístico aos dados da pesquisa. Para verificar possíveis associações entre as variáveis em estudo foram utilizados o teste qui-quadrado e o teste exato de Fisher nos casos em que as frequências esperadas foram menores que cinco, considerando o nível de significância de $5 \%(\mathrm{p}<0,05)^{(15)}$.

Em um segundo momento, construíram-se modelos de regressão logística simples e múltipla, levando em consideração diversas variáveis. Para a seleção, foi realizado o procedimento de Backward incorporando, inicialmente, todas as variáveis.

$\mathrm{Na}$ sequência, por etapas (Stepwise), foram retiradas as variáveis que possuíam maior probabilidade de não ter associação com a variável dependente (GDS). Foram consideradas, no modelo final, apenas as variáveis que apresentaram um nível de significância menor que 0,20, isto é, p-valor $<0,20$. Por fim, foram estimadas as Razões de Chances (RC), brutas e ajustadas com os respectivos intervalos de confiança (IC95\%) e com os respectivos testes de Wald. Todas as análises foram realizadas com auxílio do software $R$, ao nível de significância de 5\% (p-valor<0,05).

\section{Resultados}

Dentre os 130 entrevistados, predominaram os indivíduos do sexo feminino, faixa etária entre 60 e 69 anos com média de $\pm 71,5$ anos; residiam com o cônjuge, renda mensal de $1 \mathrm{a}$ 3 salários-mínimos, sem escolaridade, não consumiam bebidas alcoólicas, não praticavam atividade física e utilizavam o Sistema Único de Saúde (SUS) e plano de saúde privado complementar. Sintomas depressivos prevaleceram na população estudada e quatro apresentavam sintomas de depressão grave (Tabelas 1 e 2).

Tabela 1 - Distribuição das características socioeconômicos dos idosos residentes no município. Equador, Rio Grande do Norte, Brasil - 2020. (N=130)

(continua)

\begin{tabular}{|c|c|c|c|}
\hline Variáveis & $\mathbf{n}$ & $\%$ & $\mathrm{X} \wedge 2$ (p-valor) \\
\hline Gênero & & & $5,20(0,0226)$ \\
\hline Feminino & 78 & 60 & \\
\hline Masculino & 52 & 40 & \\
\hline Idade & & & $70,49(<0,001)$ \\
\hline 60 a 69 anos & 60 & 46,1 & \\
\hline 70 a 79 anos & 52 & 40 & \\
\hline 80 a 89 anos & 14 & 10,8 & \\
\hline 90 a 99 anos & 4 & 3,1 & \\
\hline Renda & & & $145,86(<0,001)$ \\
\hline < 1 salário-mínimo & 3 & 2,3 & \\
\hline 1 a 3 salários-mínimos & 107 & 82,3 & \\
\hline 4 a 5 salários-mínimos & 19 & 14,6 & \\
\hline Não declarou & 1 & 0,8 & \\
\hline Estado civil & & & $100,58(<0,001)$ \\
\hline Casado & 79 & 60,8 & \\
\hline Divorciado & 8 & 6,2 & \\
\hline Solteiro & 10 & 7,7 & \\
\hline Viúvo & 33 & 25,3 & \\
\hline Escolaridade & & & $68,58(<0,001)$ \\
\hline Sem escolaridade & 59 & 45,4 & \\
\hline 1 a 4 anos & 53 & 40,7 & \\
\hline 5 a 8 anos & 8 & 6,2 & \\
\hline$>9$ anos & 10 & 7,7 & \\
\hline
\end{tabular}


Tabela 1 - Distribuição das características socioeconômicos dos idosos residentes no município. Equador, Rio Grande do Norte, Brasil - 2020. (N=130)

(conclusão)

\begin{tabular}{|c|c|c|c|}
\hline Variáveis & $\mathbf{n}$ & $\%$ & $\mathrm{X} \wedge 2$ (p-valor) \\
\hline Serviços de saúde & & & $39,88(<0,001)$ \\
\hline Sistema Único de Saúde & 29 & 22,3 & \\
\hline Sistema Único de Saúde e Saúde Suplementar & 101 & 77,7 & \\
\hline Tabagista & & & $76,92(<0,001)$ \\
\hline Ausente & 115 & 88,5 & \\
\hline Presente & 15 & 11,5 & \\
\hline Etilista & & & $85,46(<0,001)$ \\
\hline Ausente & 117 & 90 & \\
\hline Presente & 12 & 9,2 & \\
\hline Não declarou & 1 & 0,8 & \\
\hline Atividade física & & & $17,55(0,0002)$ \\
\hline Pratica & 43 & 33,1 & \\
\hline Não pratica & 63 & 48,4 & \\
\hline Nem pratica, nem tem interesse em praticar & 24 & 18,5 & \\
\hline Doença crônica & & & $16,27(<0,001)$ \\
\hline Não & 42 & 32,3 & \\
\hline Sim & 88 & 67,7 & \\
\hline Satisfação de vida & & & $16,28(<0,001)$ \\
\hline Insatisfeito & 3 & 2,3 & \\
\hline Satisfeito & 34 & 26,2 & \\
\hline Muito satisfeito & 81 & 62,3 & \\
\hline Nem satisfeito, nem insatisfeito & 12 & 9,2 & \\
\hline \multicolumn{4}{|l|}{ Domínio mental } \\
\hline Com risco & 21 & 16,2 & \\
\hline Sem risco & 109 & 83,8 & \\
\hline \multicolumn{4}{|l|}{ Domínio físico } \\
\hline Com risco & 31 & 23,8 & $59,57(<0,001)$ \\
\hline Sem risco & 98 & 75,4 & \\
\hline Não relatou & 1 & 0,8 & \\
\hline Doenças autorreferidas & & & $34,80(<0,001)$ \\
\hline Não possui & 49 & 37,7 & \\
\hline 1 doença & 35 & 26,8 & \\
\hline 2 doenças & 34 & 26,2 & \\
\hline 3 doenças & 1 & 0,8 & $178,87(<0,001)$ \\
\hline$>3$ doenças & 11 & 8,5 & \\
\hline \multicolumn{4}{|l|}{ Uso de medicamentos } \\
\hline Não utiliza & 35 & 26,9 & \\
\hline 1 a 3 medicamentos & 64 & 49,3 & \\
\hline 4 a 5 medicamentos & 15 & 11,5 & \\
\hline$>5$ medicamentos & 16 & 12,3 & $48,52(<0,001)$ \\
\hline \multicolumn{4}{|c|}{$\begin{array}{l}\text { Geriatric Depression Scale (Escala de Depressão } \\
\text { Geriátrica) }\end{array}$} \\
\hline 0 a 5 pontos & 48 & 36,9 & \\
\hline 6 a 10 pontos & 78 & 60 & \\
\hline 11 a 15 pontos & 4 & 3,1 & \\
\hline Total & 130 & 100 & $63,94(<0,001)$ \\
\hline
\end{tabular}

Fonte: Elaboração própria.

Nota: Salário-Mínimo vigente na época da coleta de dados $=\mathrm{R} \$ 1.045,00$. 
Verificou-se que os fumantes declararam uma média de pelo menos três cigarros por dia, nos últimos 30 dias; a quase metade praticava caminhada e outras atividades, como andar de bicicleta e pilates em uma média de 30 minutos, cerca de três vezes na semana nos últimos sete dias; alguns referiram participar regularmente de alguma atividade de lazer ou atividade física esporadicamente. Por fim, houve o registro de apresentar limitações na marcha, isto é, andavam com algum tipo de suporte ou tinham dificuldade em executar movimentos de precisão com controle e destreza. Outros apresentaram risco quanto ao domínio mental. A maioria dos participantes relatou algum tipo de doença crônica, destacando-se Hipertensão Arterial Sistêmica, Diabetes mellitus e Osteoartrites. Destaca-se que a maior parte da amostra fazia uso de medicamentos diariamente, ingerindo de um a três tipos; também foram citados o uso de três a cinco e de mais de cinco fármacos diários.

Tabela 2 - Análises bruta e ajustada para fatores associados à ocorrência de sintomas depressivos segundo a Escala de Depressão Geriátrica Reduzida entre idosos do município. Equador, Rio Grande do Norte, Brasil - 2020. (N=130)

\begin{tabular}{|c|c|c|c|}
\hline Variáveis & $\begin{array}{c}\text { Razão de Chances bruta } \\
\text { (Intervalo de Confiança 95\%) }\end{array}$ & $\begin{array}{c}\text { Razão de Chances } \\
\text { ajustada (Intervalo de } \\
\text { Confiança 95\%) }\end{array}$ & p-valor \\
\hline \multicolumn{4}{|c|}{ Está satisfeito(a) com sua vida? } \\
\hline $\operatorname{Sim}$ & $0,4(0,12 ; 1,42)$ & 0,158 & 0,167 \\
\hline Não & $1,00-$ & 1,00 & - \\
\hline \multicolumn{4}{|c|}{ Diminuiu a maior parte de suas atividades e interesses? } \\
\hline Sim & $1,00-$ & 1,00 & - \\
\hline Não & $11(4,12 ; 29,35)$ & $<0,001$ & $<0,001$ \\
\hline \multicolumn{4}{|c|}{ Sente que a vida está vazia? } \\
\hline Sim & 1,00 & 1,00 & - \\
\hline Não & - & - & - \\
\hline \multicolumn{4}{|c|}{ Aborrece-se com frequência? } \\
\hline Sim & 1,00 & 1,00 & - \\
\hline Não & $18,95(2,48 ; 144,63)$ & 0,005 & $<0,001$ \\
\hline \multicolumn{4}{|c|}{ Sente-se de bem com a vida na maior parte do tempo? } \\
\hline $\operatorname{Sim}$ & $0,18(0,07 ; 0,44)$ & $<0,001$ & $<0,001$ \\
\hline Não & 1,00 & 1,00 & - \\
\hline \multicolumn{4}{|c|}{ Teme que algo ruim possa lhe acontecer? } \\
\hline $\operatorname{Sim}$ & 1,00 & 1,00 & - \\
\hline Não & $13,9(4,52 ; 42,73)$ & $<0,001$ & $<0,001$ \\
\hline \multicolumn{4}{|c|}{ Sente-se feliz a maior parte do tempo? } \\
\hline $\operatorname{Sim}$ & $0,12(0,04,0,43)$ & 0,001 & $<0,001$ \\
\hline Não & 1,00 & 1,00 & - \\
\hline \multicolumn{4}{|c|}{ Sente-se frequentemente desamparado? } \\
\hline $\operatorname{Sim}$ & 1,00 & 1,00 & - \\
\hline Não & - & - & - \\
\hline \multicolumn{4}{|c|}{ Prefere ficar em casa a sair e fazer coisas novas? } \\
\hline $\operatorname{Sim}$ & 1,00 & 1,00 & - \\
\hline Não & $3,59(1,6 ; 8,05)$ & 0,002 & 0,002 \\
\hline \multicolumn{4}{|c|}{ Acha que tem mais problemas de memória que a maioria? } \\
\hline $\operatorname{Sim}$ & 1,00 & 1,00 & - \\
\hline Não & $4,97(2,15 ; 11,46)$ & $<0,001$ & $<0,001$ \\
\hline \multicolumn{4}{|c|}{ Acha que é maravilhoso estar vivo agora? } \\
\hline Sim & $0,7(0,24,2,03)$ & 0,51 & 0,517 \\
\hline Não & 1,00 & 1,00 & - \\
\hline
\end{tabular}


Tabela 2 - Análises bruta e ajustada para fatores associados à ocorrência de sintomas depressivos segundo a Escala de Depressão Geriátrica Reduzida entre idosos do município. Equador, Rio Grande do Norte, Brasil - 2020. ( $N=130)$

(conclusão)

\begin{tabular}{|c|c|c|c|}
\hline Variáveis & $\begin{array}{c}\text { Razão de Chances bruta } \\
\text { (Intervalo de Confiança 95\%) }\end{array}$ & $\begin{array}{c}\text { Razão de Chances } \\
\text { ajustada (Intervalo de } \\
\text { Confiança 95\%) }\end{array}$ & p-valor \\
\hline \multicolumn{4}{|c|}{ Vale a pena viver como vive agora? } \\
\hline $\operatorname{Sim}$ & $1,4(0,48 ; 4,12)$ & 0,538 & 0,529 \\
\hline Não & 1,00 & 1,00 & - \\
\hline \multicolumn{4}{|c|}{ Sente-se cheio(a) de energia? } \\
\hline Sim & $1,09(0,45 ; 2,63)$ & 0,85 & 0,85 \\
\hline Não & 1,00 & 1,00 & - \\
\hline \multicolumn{4}{|c|}{ Acha que sua situação tem solução? } \\
\hline $\operatorname{Sim}$ & $6,51(1,86 ; 22,81)$ & 0,003 & $<0,001$ \\
\hline Não & 1,00 & 1,00 & - \\
\hline \multicolumn{4}{|c|}{ Acha que tem muita gente em situação melhor? } \\
\hline Sim & $4,68(1,04,21,13)$ & 0,045 & 0,018 \\
\hline Não & 1,00 & 1,00 & - \\
\hline
\end{tabular}

Fonte: Elaboração própria.

Notas: RC é a razão de chances de o evento "desenvolver a doença" entre indivíduos expostos dividida pela razão de chances de doença entre não-expostos.

Sinal convencional utilizado:

- Dado numérico igual a zero não resultante de arredondamento.

$\mathrm{Na}$ análise, todas as variáveis apresentaram associação com os sintomas depressivos. No $\mathrm{x}$ valor, a prevalência de sintomas depressivos entre as mulheres foi quase duas vezes maior do que entre os homens (p-valor (5,20-0,0226)). Eles apresentaram um incremento linear na associação com autopercepção de saúde, isto é, aqueles com autopercepção maior que três doenças (p-valor 178,87 (<0,001)) apresentaram as maiores prevalências de sintomas depressivos quando comparados aos idosos com avaliação de uma ou duas comorbidades de saúde.

\section{Discussão}

A depressão em idosos é um sério e crescente problema de saúde pública, trazendo prejuízos à vida familiar e à comunidade. Não é condição natural do envelhecimento e muito menos uma característica de fraqueza; é uma doença, e deve ser reconhecida e tratada efetivamente, para o desenvolvimento físico e mental dessa população ${ }^{(16)}$.

Os achados sociodemográficos deste estudo estão em conformidade com a literatura nos aspectos gênero feminino e renda familiar ${ }^{(16-17)}$.
Ainda quanto ao gênero feminino, outros estudos analisados também demonstraram resultados semelhantes ${ }^{(18-20)}$. Este fato está de acordo com dados da literatura, que sugerem a vulnerabilidade das mulheres ao desenvolvimento de sintomas depressivos na velhice. Dentre as possíveis explicações está o fato de que elas vivem, em média, mais do que os homens. Além disso, idades mais avançadas são acompanhadas por maior incidência de doenças crônicas, como a depressão ${ }^{(21)}$.

Entende-se que o maior risco de depressão em mulheres pode decorrer da sobrecarga de suas funções sociais (esposa, mãe, cuidadora, educadora, entre outras), da maior taxa de viuvez e sobrevida, do isolamento social e da privação de estrogênio ${ }^{(19-22)}$.

No que diz respeito à situação conjugal, a condição de não ter companheiro mostrou-se associada à presença de sintomas depressivos na literatura, enquanto, neste estudo, foi prevalente nos casados. Esse acontecimento pode ter ocorrido pelo fato de a mulher ter sua vida cercada de limitações, pelo desejo e/ou imposição da família e da sociedade ${ }^{(19-22)}$ : não estudar nem trabalhar, se o marido não permitir, não querer 
ter mais filhos, não poder se separar, simplesmente não poder ser. Ressalta-se que a história da família no Brasil tem como base o patriarcado, sistema que traz arraigado o conceito de dominação masculina sobre as mulheres. Essa tradição perpetua-se, mesmo que simbolicamente, e causa, por vezes, sintomas depressivos ${ }^{(19-22)}$.

O presente estudo, portanto, encontrou uma ligação significativa entre sintomas depressivos e o idoso na faixa etária dos 60 aos 69 anos, casados com mais de nove anos de escolaridade, baixa renda e hábitos tabagista e etilista ausentes. Estes achados diferenciam-se dos encontrados em outros estudos brasileiros realizados com idosos, hospitalizados ou residentes na comunidade ${ }^{(22-25)}$.

Acredita-se que o nível educacional possibilita ao indivíduo ampliação de recursos no enfrentamento das situações estressantes da vida, o que não condiz, em parte, com os achados da pesquisa no tocante aos indivíduos analfabetos, que apresentariam maior risco de desenvolver sintomas depressivos ${ }^{(25)}$.

O número de idosos analfabetos e de baixa escolaridade é bastante significativo no Brasil, sobremaneira quando se refere à região Nordeste, com impactos negativos em diversos aspectos da qualidade de vida. Nesta investigação, esse fator diferenciou-se do encontrado em estudos que mostraram a significância desse elemento, o que contesta a afirmação de que a escolaridade seja considerada um fator de proteção nesses $\operatorname{aspectos}^{(21-23)}$.

O valor do SM no Brasil, geralmente, é insuficiente para custear as necessidades do idoso $^{(24)}$. Assim, alguns deles ainda desenvolvem atividades no dia a dia, com o intuito de obter renda extra, para complementar o salário que não contempla suas necessidades e as de seus familiares. Isto ocorre porque, muitas vezes, é o idoso quem sustenta a família ${ }^{(24-25)}$.

Quanto à prática de atividade física, sabe-se que, quando realizada regularmente, confere proteção contra a ocorrência de depressão ${ }^{(25)}$. Há evidência suficiente dos efeitos benéficos da atividade física sobre a depressão em pessoas idosas. Um estudo controlado randomizado mostrou que, ao se sentir menos deprimido, o indivíduo mostra-se mais propenso a manter-se fisicamente ativo e diminui a probabilidade de retorno dos sintomas depressivos ${ }^{(26)}$.

No que se refere à utilização de serviços de saúde, os entrevistados referiram buscá-los somente quando necessário. Isso demonstra que muitos idosos não possuem acompanhamento em saúde, procurando atendimento apenas em momentos críticos ou no aparecimento de sintomas agudos. Mostra ainda a ineficiência das buscas ativas, que são relevantes na APS, além de apontarem as dificuldades de acesso dos idosos às UBS, a falta de visitas domiciliares, entre outros aspectos ${ }^{(24)}$.

Pelo fato de não possuir vínculos apenas com planos de saúde e não frequentar serviços de APS, as ações e medidas de promoção à saúde e prevenção de doenças tornam-se distantes desse público, assim como a realização de diagnósticos e intervenções precoces. Quando se fala em saúde mental e depressão, a falta de acompanhamento e tratamento podem levar ao agravamento da condição de saúde. No caso do idoso, traz danos, tais como o surgimento de doenças crônicas e o risco de suicídio ${ }^{(24)}$.

Um fator importante nos idosos com potenciais riscos para o desenvolvimento de sintomas depressivos foi o risco no domínio mental e físico, além de os idosos com mais de três problemas de saúde autorreferidos fazerem uso de mais de cinco medicações. Este resultado condiz com o encontrado em outros estudos ${ }^{(21-25)}$.

Sabe-se que a etiologia da depressão no idoso é multifatorial, podendo envolver fatores genéticos, eventos vitais, como luto e abandono, e doenças incapacitantes. Os distúrbios depressivos podem ocasionar indiferença ao meio ambiente, alteração do nível de atenção e da deambulação, diminuição de energia e autoconfiança, reclusão e perdas cognitivas ${ }^{(21-25)}$. Desse modo, o uso rotineiro, por parte dos profissionais de saúde, de instrumentos de triagem para depressão, facilmente aplicáveis na prática clínica, permitiria não só o diagnóstico de muitos casos da doença como também o prognóstico das comorbidades existentes, as quais contribuem para 
maior mortalidade, seja pelo aumento do risco de suicídio, seja pela evolução desfavorável das doenças crônicas existentes ${ }^{(25)}$.

Como limitação técnica de operacionalização do estudo, cita-se a dificuldade de localização dos idosos selecionados, conforme o procedimento amostral, sobretudo na zona rural, que foi inviabilizada devido à pandemia por COVID-19. No entanto, com a efetiva colaboração dos agentes comunitários de saúde (ACS) do município, essa dificuldade foi progressivamente contornada.

Este estudo contribuiu de forma eficaz para o alcance dos objetivos propostos e direcionou as ações das autoridades de saúde do município em questão para o combate à depressão. Verificou-se que, após a divulgação dos resultados da pesquisa, foram realizados investimentos na oferta de capacitações das equipes de Estratégia Saúde da Família, bem como na contratação de psiquiatra e psicólogos para compor a equipe especializada. Enfim, entendeu-se a necessidade de atender a alta demanda local, no tocante aos aspectos biopsicossociais, contribuindo, assim, para o bem-estar e a qualidade de vida da população.

\section{Conclusão}

A prevalência de sintomas depressivos, identificada neste estudo, foi alta e alerta para a necessidade de maiores cuidados de saúde com a população idosa do munícipio estudado. Especial atenção deve ser dada àqueles idosos que apresentam percepção negativa da saúde, que não fazem atividade física regular, que apresentam utilização de mais de cinco medicamentos, mulheres, casados e com salários entre 1 e 3 salários-mínimos. A prevalência desses sintomas na população requer investimentos em ações de promoção de saúde, atentando para as práticas que estimulem o envelhecimento ativo e diminuam a incapacidade funcional.

O delineamento transversal utilizado no presente estudo possibilitou identificar a prevalência de sintomas depressivos em amostra representativa dos idosos atendidos na APS.
Todavia, destaca-se a possibilidade de causalidade reversa como, por exemplo, nas associações do desfecho com autoavaliação de saúde e satisfação com a vida. Optou-se por mantê-las na análise, por serem relevantes para o planejamento das ações em saúde relacionadas ao cuidado da população idosa.

Recomenda-se a utilização do instrumento GDS-15 na rotina dos serviços de APS, de modo a orientar a intervenção dos profissionais de saúde. Sugere-se, desta forma, que outros estudos sejam idealizados e executados, no que tange à utilização do instrumento nos idosos, de modo a favorecer a robustez do conhecimento científico nessa área.

\section{Colaborações:}

1 - concepção, projeto, análise e interpretação dos dados: Evanilza Maria Marcelino, Ricardo Alves de Olinda e Ana Claudia Torres de Medeiros;

2 - redação do artigo e revisão crítica relevante do conteúdo intelectual: Evanilza Maria Marcelino, Priscila Maria de Castro Silva, Fabíola de Araújo Leite Medeiros, Josefa Raquel Luciano da Silva, Ricardo Alves de Olinda e Ana Claudia Torres de Medeiros;

3 - aprovação final da versão a ser publicada: Evanilza Maria Marcelino e Ana Claudia Torres de Medeiros.

\section{Referências}

1. Lacerda MA, Silva LLT, Oliveira F, Coelho KR. O cuidado com o idoso fragilizado e a Estratégia Saúde da Família: perspectivas do cuidador informal familiar. Rev baiana enferm. 2021;35:e43127. DOI: https://doi.org/10.18471/ rbe.v35.43127

2. Figueiredo AEB, Ceccon RF, Figueiredo JHC. Doenças crônicas não transmissíveis e suas implicações na vida de idosos dependentes. Ciênc Saúde Colet. 2021;26(1):77-88. DOI: https:// doi.org/10.1590/1413-81232020261.33882020

3. Silva RS, Fedosse E, Pascotini FS, Riehs EB. Condições de saúde de idosos institucionalizados: contribuições para ação interdisciplinar e 
promotora de saúde. Cad Bras Ter Ocup. 2019;27(2):345-56. DOI: https://doi.org/10.4322/ 2526-8910.ctoAO1590

4. Oliveira DV, Pivetta NRS, Oliveira GVN, Silva DA, Nascimento JJRA, Cavaglieri CR. Fatores intervenientes nos indicativos de depressão em idosos usuários das unidades básicas de saúde de Maringá, Paraná, 2017. Epidemiol Serv Saúde. 2019;28(3):1-10. DOI: https://doi.org/10.5123/s1679-49742019000300010

5. Sousa KA, Freitas FFQ, Castro AP, Oliveira CDB, Almeida AAB, Sousa KA. Prevalência de sintomas de depressão em idosos assistidos pela Estratégia de Saúde da Família. REME - Rev Min Enferm. 2017;21:e-1018. DOI: https://doi. org/10.5935/1415-2762.20170028

6. Bhering AC, Amaral Júnior JC, Pio II JM, Rodrigues I, Valadão AF, Quintão MAU. Distúrbios psíquicos menores e fatores associados em um grupo de idosos. Revista UNINGÁ [Internet]. 2017 [cited 2019 Oct 10];53(1):12-8. Available from: http://revista.uninga.br/index.php/uninga/article/ view/1419

7. Gullich I, Duro SMS, Cesar JA. Depressão entre idosos: um estudo de base populacional no Sul do Brasil. Rev bras epidemiol. 2016;19(4):691-701. DOI: https://doi.org/10.1590/1980-549720160004 0001

8. Silva PAS, Rocha SV, Santos LB, Santos CA, Amorim CR, Vilela ABA. Prevalência de transtornos mentais comuns e fatores associados entre idosos de um município do Brasil. Ciênc. saúde colet. 2018;23(2):639-46. DOI: https://doi. org/10.1590/1413-81232018232.12852016

9. Murcho N, Pacheco E, Jesus SN. Transtornos mentais comuns nos Cuidados de Saúde Primários: um estudo de revisão. Rev Portuguesa Enferm Saúde Mental. 2016;(15):30-6. DOI: http:// dx.doi.org/10.19131/rpesm.0129

10. Onofri Junior VA, Martins VS, Marin MJS. Atenção à saúde do idoso na Estratégia Saúde da Família e a presença de transtornos mentais comuns. Rev bras geriatr gerontol. 2016;19(1):2133. DOI: $10.1590 / 1809-9823.2016 .15004$

11. Brasil. Conselho Nacional de Saúde. Resolução n. 466, de 12 de dezembro de 2012. Aprova diretrizes e normas regulamentadoras de pesquisas envolvendo seres humanos [Internet]. Brasília (DF); 2012 [cited 2021 Oct 25]. Available from: https://conselho.saude.gov.br/resolucoes/2012/ Reso466.pdf
12. Bertolucci PHF, Brucki SMD, Campacci SR, Juliano Y. O Mini-Exame do Estado Mental em uma população geral: impacto da escolaridade. Arq Neuro-Psiquiatr. 1994;52(1):1-7. DOI: https://doi. org/10.1590/S0004-282X1994000100001

13. Sheikh JI, Yesavage JA. Geriatric Depression Scale (GDS): Recent Evidence and Development of a Shorter Version. Clin Gerontol. 1986;5(1-2):165-73. DOI: https://doi.org/10.1300/J018v05n01_09

14. Almeida OP, Almeida SA. Confiabilidade da versão brasileira da Escala de Depressão em Geriatria (GDS) versão reduzida. Arq Neuro-Psiquiatr. 1999;57(2B):421-6. DOI: https:// doi.org/10.1590/S0004-282X1999000300013

15. Siegel S, Castellan JNJ. Estatística não-paramétrica para ciências do comportamento. 2a ed. São Paulo: Bookman (Artmed); 2006.

16. Scherrer Júnior G, Okuno MFP, Oliveira LM, Barbosa DA, Alonso AC, Fram DS, et al. Quality of life of institutionalized aged with and without symptoms of depression. Rev Bras Enferm. 2019;72(Suppl 2):127-33. DOI: https://doi. org/10.1590/0034-7167-2018-0316

17. Ribeiro DS, Garbin K, Jorge MSG, Doring M, Portella MR, Wibelinger LM. Prevalence of chronic pain and analysis of handgrip strength in institutionalized elderly. BrJP. 2019;2(3):242-6. DOI: http://dx.doi.org/10.5935/2595-0118.20190043

18. Sigström R, Waern M, Gudmundsson P, Skoog I, Östling S. Depressive spectrum states in a population-based cohort of 70-year olds followed over 9 years. Int J Geriatr Psychiatry. 2018;33(8):1028-37. DOI: https://doi.org/10.1002/ gps. 4888

19. Isaac L, Ferreira CR, Ximenes VS. Cuidar de idosos: um assunto de mulher? Estud Interdiscip Psicol. 2018;9(1):108-25. DOI: https://doi. org/10.5433/2236-6407.2016v9n1p108

20. Santos EB, Rodrigues RAP, Pontes-Neto OM. Prevalence and predictors of post stroke depression among elderly stroke survivors. Arq Neuropsiquiatr. 2016;74(8):621-5. DOI: https://doi. org/10.1590/0004-282X20160088

21. Gullich I, Duro SMS, Cesar JA. Depressão entre idosos: um estudo de base populacional no Sul do Brasil. Revbras epidemiol. 2016;19(4):691-701. DOI: https://doi.org/10.1590/1980-5497201600040001

22. Guimaraes LA, Brito TA, Pithon KR, Jesus CS, Souto CS, Souza SJN, et al. Sintomas depressivos e fatores associados em idosos residentes em 
instituição de longa permanência. Ciênc Saúde Colet. 2019;24(9):3275-82. DOI: https://doi. org/10.1590/1413-81232018249.30942017

23. Maximiano-Barreto MA, Fermoseli AFO. Prevalência de ansiedade e depressão em idosos de baixa escolaridade em Maceió/Al. Psicol saúde doenças. 2017;18(3):801-13. DOI: http://dx.doi. org/10.15309/17psd180314

24. Güths JFS, Jacob MHVM, Santos AMPV, Arossi GA, Béria JU. Perfil sociodemográfico, aspectos familiares, percepção de saúde, capacidade funcional e depressão em idosos institucionalizados no Litoral Norte do Rio Grande do Sul,
Brasil. Rev bras geriatr gerontol. 2017;20(2):175-85. DOI: https://doi.org/10.1590/1981-22562017020. 160058

25. Shin C, Park MH, Lee S-H, Ko Y-H, Kim Y-K, Han K-M, et al. Usefulness of the 15-item geriatric depression scale (GDS-15) for classifying minor and major depressive disorders among community-dwelling elders. J Affect Disord. 2019; 259:370-5. DOI: https://doi.org/10.1016/j.jad.2019. 08.053

Recebido: 24 de agosto de 2021 Aprovado: 8 de novembro de 2021

Publicado: 7 de dezembro de 2021

A Revista Baiana de Enfermagem utiliza a Licença Creative Commons - Atribuição-NãoComercial 4.0 Internacional. https://creativecommons.org/licenses/by-nc/4.0/

Este artigo é de acesso aberto distribuído sob os termos da Licença Creative Commons (CC BY-NC).

Esta licença permite que outros remixem, adaptem e criem a partir do seu trabalho para fins não comerciais. Embora os novos trabalhos tenham de lhe atribuir o devido crédito e não possam ser usados para fins comerciais, os usuários não têm de licenciar esses trabalhos derivados sob os mesmos termos. 